\title{
Study on Streptococcus Pneumoniae Diseases in Chi- na : the Past, Present and Future
}

\author{
楊永弘 (Yang Yonghong) \\ (Beijing Children's Hospital Affilated to Capital University of Medical Sciences)
}

Invasive infection caused by Streptococcus pneumoniae (S.p) is a common and serious disease among children, for which WHO-certified conjugate vaccines are currently available and strongly recommended for universal infant immunization. Before these vaccines to be used in China, it is neccessary to have a comprehensive data and clear understanding in regards of the disease burden, including incidence, age distribution and clinical outcome of S.p meningitis and other systemic infections in this region.

Data on S.p disease in China are still scant at this moment and it is particularly so before 1980 with only a few literatures related assessable. S.p meningitis once spread widely across China in 1950s-1960s and accounted for up to $11.1 \% \sim 22.1 \%$ of all acute bacterial meningitis. It was the second major cause of the disease, following meningococcus. In the meantime, up to $38 \%$ of bacterial meningitis cases were caused by unknown pathogens in despite of the severe epidemic of meningococcal meningitis during that period. Regrettably, exact data regarding pathogens of children's pneumonia during that period is not available.

Since 1980s, researchers have been conducting hospital- and community-based etiologic and epidemiologic studies on bacterial meningi- tis in China. Findings of these studies suggest that S.p occupied $8 \%$ to $21.0 \%$ of bacterial meningitis in total. To take Hefei city (1990-1992) as the example, incidence of S.p meningitis in that City was 1.5 per 100000 Children $<5$ years. Moreover, the studies indicated that pneumonia was the primary cause of death for the Chinese children. From 1991 to 1993, the average mortality rate of children $<5$ years because of pneumonia reached 1563.2 per 100000 in China, with about $16.7 \%$ lower respiratory infection caused by S.p. Furthermore, a issue of particular concern arising from these studies was the severity of the armicrobial resistance of S.p. In addition, the serogroups/serotypes of S.p vary with research area, timing and the samples' disease as well as their ages. It is worth noting that the 7 values S.p vaccine widely applied in America and Europe can only cover less than $50 \%$ of S.p infection cases in some study. $\mathrm{Nev}^{-}$ ertheless, S.p vaccination for infants and children can truly be considered as an effective and valuable approach to reduce the incidence. We should therefore work to carry out a national survey and continuous monitoring regarding the situation of S.p infection diseases among children so as to acquire a set of precise and comprehensive information. 